TRANSACTIONS OF THE

AMERICAN MATHEMATICAL SOCIETY

Volume 172, October 1972

\title{
VECTOR VALUED ABSOLUTELY CONTINUOUS FUNCTIONS ON IDEMPOTENT SEMIGROUPS
}

\author{
BY
}

\author{
RICHARD A. ALÒ, ANDRE DE KORVIN AND RICHARD J. EASTON
}

\begin{abstract}
In this paper the concept of vector valued, absolutely continuous functions on an idempotent semigroup is studied. For $F$ a function of bounded variation on the semigroup $S$ of semicharacters with values of $F$ in the Banach space $X$, let $A=\operatorname{AC}(S, X, F)$ be all those functions of bounded variation which are absolutely continuous with respect to $F$. A representation theorem is obtained for linear transformations from the space $A$ to a Banach space which are continuous in the BV-norm. A characterization is also obtained for the collection of functions of $A$ which are Lipschitz with respect to $F$. With regards to the new integral being utilized it is shown that all absolutely continuous functions are integrable.
\end{abstract}

Introduction. Absolutely continuous functions have been extensively studied in the literature. For example in [5] the dual space of the space of absolutely continuous functions is characterized. In [7], T. Hildebrandt gives a representation theorem for the linear functionals on $\mathrm{BV}[0,1]$ which are continuous in the weak topology. In [6] a representation theorem for linear functionals continuous in the variation norm on $\mathrm{BV}[0,1]$ is given. This representation is in terms of a socalled $v$-integral. The techniques of that paper, however, make strong use of the order on $[0,1]$. In [8] absolutely continuous functions and functions of bounded variation on idempotent semigroups are defined and these functions are identified with a certain class of finitely additive set functions.

In [1], the identification in [8] is used to obtain a representation theorem. A characterization of the so-called Lipschitz functions in the setting of [8] is also obtained by the authors. The techniques of [1] depend on a result of Darst [2] which states that if $u$ and $v$ are two finitely additive real valued set functions with $v \ll<$, then $v$ is the limit in the variation norm of finitely additive set functions $u_{n}$ defined by $u_{n}(A)=\int_{A} s_{n} d u$, where $s_{n}$ is a simple function. This result does not in general hold true when $u$ and $v$ are vector valued.

In this paper we study the concept of vector valued, absolutely continuous

Received by the editors January 26, 1972.

$A M S(M O S)$ subject classifications (1970). Primary 46G10, 46E40; Secondary 28A25, 28 A 45.

Key words and phrases. Bounded variation, absolutely continuous, v-integral, Banach space, linear transformation, dual space, Lipschitz functions, convex set functions, semicharacter, semigroup, set-functions, Möbius function, positive definite, polygonal function, characteristic function, simple function. 
functions on an idempotent semigroup. We obtain a representation theorem for linear transformations from the space $A C(S, X, F)$ to $Y$ which are continuous in the BV-norm. A characterization is also obtained for the collection of functions of $\operatorname{AC}(S, X, F)$ which are Lipschitz with respect to $F$. It is also shown that this new integral being utilized has a "wide enough" class of integrable functions. In fact all polygonal functions are integrable (see Lemma 6) and, even more so, all absolutely continuous functions (Theorem 2) are integrable.

1. Notations and definitions. Let $A$ be an abelian idempotent semigroup, and let $S$ be a semigroup of semicharacters on $A$ containing the identity. Recall that a semicharacter on $A$ is a nonzero bounded, complex valued function on $A$ which is a semigroup homomorphism. Since $A$ is idempotent it is clear that every $f$ in $S$ can be viewed as a characteristic function on $A$. The notations used here will be consistent with the ones used in [1] and [8]. We recall some of these notations.

For $f$ in $S, A_{f}=\{a \in A: f(a)=1\}$ and $J_{f}=\{a \in A: f(a)=0\}$. Let $T_{n}$ be the set of all $n$-tuples consisting of 0 and 1 . Let $Q_{n}$ be a finite subset of $S$, that is $Q_{n}=\left\{f_{1}, f_{2}, \cdots, f_{n}\right\}$, and let $\sigma \in T_{n}$. If $\sigma(i)$ denotes the $i$ th component of $\sigma$, for $Q=Q_{n}$ let

$$
B(Q, \sigma)=\left(\bigcap_{\sigma(i)=1} A_{f_{i}} \cap \bigcap_{\sigma(i)=0} J_{f_{i}}\right)
$$

Any set of this form will be called a set of $B$-type. Let $F$ be any function from $S$ to the reals. Define

$$
L(Q, \sigma) F=\sum_{\tau \in T_{n}} m(\sigma, \tau) F\left(\prod_{i=1}^{n} f_{i}^{\tau(i)}\right)
$$

where $m$ denotes the Möbius function for $T_{n}$ (see [9]). The function $F$ is said to be of bounded variation if sup $\Sigma_{\sigma \in T_{n}}|L(Q, \sigma) F|<\infty$, where the supremum is taken over all partitions of $A$ into sets $B(Q, \sigma)$ as $\sigma$ ranges over $T_{n^{*}}$. The collection of all real valued functions of bounded variation on $S$ will be denoted by $\mathrm{BV}(S)$. Consider $F \in \mathrm{BV}(S)$. Then by $\mathrm{AC}(S, F)$, we mean all functions $G \in \mathrm{BV}(S)$ such that, for each $\epsilon>0$, there exists a $\delta>0$ such that, for every finite set $Q=$ $Q_{n}$ of $S$ and any subset $H$ of $T_{n}$,

$$
\sum_{\sigma \epsilon H}|L(Q, \sigma) G|<\epsilon \quad \text { if } \sum_{\sigma \in H}|L(Q, \sigma) F|<\delta .
$$

From now on $F$ will be assumed to be positive definite, i.e. $L(Q, \sigma) F \geq 0$ for all such $Q$ and $\sigma$.

Let $X$ be a Banach space. Then by the space $\operatorname{BV}(S, X)$ we mean all functions from $S$ to $X$ which are of bounded variation in the above sense where abso- 
lute value is replaced by the norm in $X$. For $G \in \mathrm{BV}(S, X),\|G\|_{\mathrm{BV}}$ will denote $\sup \Sigma_{\sigma \in T_{n}}\|L(Q, \sigma) G\|_{X^{*}}$

Definition. Let $\Sigma$ be any field of subsets of some set and let $u$ and $v$ be finitely additive set functions defined on $\Sigma$ where $u$ is scalar valued and $v$ is $X$ valued. We say that $v$ is absolutely continuous with respect to $u$ and write $v \ll u$ if $v$ is the limit in the variation norm of $X$ valued set functions of the form $\sum_{i=1}^{n} v_{i} \cdot x_{i}$, where $x_{i} \in X$, and each $v_{i}$ is a scalar valued, finitely additive, set function on $\Sigma$, which is $\epsilon-\delta$ absolutely continuous with respect to $u$.

Remark. In the case that $X$ is the reals the above definition reduces to the usual one.

Definition. Consider $G \in \mathrm{BV}(S, X)$ and $F$ as above. The function $G$ is called absolutely continuous with respect to $F$ if $G$ is the limit in $\|\cdot\|_{\mathrm{BV}}$ of $X$ valued functions of the form $\sum_{i=1}^{n} G_{i} \cdot x_{i}$ where $x_{i} \in X$ and each $G_{i}$ is a scalar valued function defined on $S$ which is absolutely continuous with respect to $F$ as in [8]. We denote this space by $\operatorname{AC}(S, F, X)$.

2. Results. Let $u$ denote a scalar valued finitely additive set function defined on $\Sigma$ and let $m$ be an $X$-valued finitely additive set function defined on $\Sigma$.

Lemma 1. $m<u$ if and only if $m$ is the limit in the variation norm of finitely additive $X$-valued set functions defined on $\Sigma$ whose range is finite dimensional, and which are $\epsilon-\delta$ absolutely continuous with respect to $u$.

Proof. Suppose that $m$ is the limit in the variation norm of finitely additive set functions $m_{i}$ (where the ranges are finite dimensional) which are $\epsilon-\delta$ absolutely continuous with respect to $u$. It follows that each $m_{i}$ can be written as

$$
m_{i}=\sum_{j=1}^{n_{i}} m_{i j} \cdot x_{i j}
$$

where each $m_{i j}$ is a finitely additive, real valued, set function defined on $\Sigma$ each of which are $\epsilon-\delta$ absolutely continuous with respect to $u$, and where the $x_{i j}$ are linearly independent. Hence $m<<u$. The converse is clear.

Lemma 2. $m \ll u$ if and only if $m$ is the limit in the variation norm of $X-v a l$ ued set functions which are represented by integrals of $X$-valued simple functions with respect to $u$.

Proof. From Lemma $1, m \ll u$ if and only if. $m$ is the limit in the variation norm of set functions of the form $\Sigma m_{i} \cdot x_{i}$ where each $m_{i}$ is real valued and $\epsilon-\delta$ absolutely continuous with respect to $u$. From a result due to Darst [1], each $m_{i}$ is the limit in the variation norm of set functions of the form $\int s_{i, k} d u$ where each $s_{i, k}$ is a real valued simple function. 
It is clear then that $m$ will be approximated in the variation norm by $\int \Sigma s_{i, k} \cdot x_{i} d u$.

From now on $\Sigma$ will denote the field generated by all $J_{f}$ as $f$ ranges over $S$.

Let $m$ be a finitely additive $X$-valued set function defined on $\Sigma$. To $m$ we associate an $X$-valued function defined on $S$, denoted by $\hat{m}$, which is defined by

$$
\hat{m}(f)=\int f d m=m\left(A_{f}\right) .
$$

Let $\operatorname{BV}(\Sigma, X)$ denote the collection of all finitely additive $X$-valued set functions of bounded variation. Then $\operatorname{BV}(\Sigma, X)$ is a Banach space under the variation norm $[5]$.

Theorem 1. The map $m \rightarrow \hat{m}$ is a linear isometry from $\mathrm{BV}(\Sigma, X)$ onto $\mathrm{BV}(S, X)$. Moreover, $m<<u$ if and only if $\hat{m}<<\hat{u}$ and, for eacb $x$ in $X, \widehat{u \cdot x}=$ $\hat{u} \cdot x$.

Proof. Clearly the map is linear, we now show that it is onto. Consider $G \epsilon$ $\operatorname{BV}(S, X)$, then $G$ can be extended to the linear span of $S$ by the equation $G\left(\Sigma a_{i} f_{i}\right)=\Sigma a_{i} G\left(f_{i}\right)$, since $S$ is a linearly independent set (see [7, Lemma 1.4]). Since $S$ is a semigroup, for each $E \in \mathbb{Q}$, it follows that $\chi_{E}$ is an element of the linear span of $S$. Thus we define a set function $u_{G}$ by equation $u_{G}(E)=G\left(\chi_{E}\right)$. It follows that $u_{G}$ is a finitely additive $X$-valued set function defined on $\Sigma$. Furthermore for each $f \in S, \hat{u}_{G}(f)=u_{G}\left(A_{f}\right)=G(f)$. We now show that the map is norm preserving. We have $\|m\|=\sup \Sigma\left\|m\left(B_{i}\right)\right\|$ where the $B_{i}$ 's are sets of $B$-type and form a partition of $A$. Now

$$
\|m\|=\sup \sum\left\|m\left(B_{i}\right)\right\|=\sup \sum\left\|L\left(B_{i}\right) \hat{m}\right\|=\|\hat{m}\|_{\mathrm{B} \mathrm{V}} .
$$

Note that we can now obtain the norm of $G$ directly from the equation $\|G\|_{\mathrm{B} \mathrm{V}}=$ sup $\Sigma\left\|G\left(\chi_{A}\right)\right\|$. Now suppose that $G<<F$. Then $G$ is the limit in the variation norm of $G_{n}=\Sigma_{i} b_{n, i} \cdot x_{n, i}$ where each $b_{n, i}$ is real valued and $b_{n, i}<<F$. Also each $b_{n, i}=\hat{u}_{n, i}$ where $u_{n, i}<<u_{F}$. Hence if we let $u_{n}=\Sigma_{i} u_{n, i} \cdot x_{n, i}$ it follows that $u_{n}$ converges to $u_{G}$ in the variation norm once we have shown that, for each real valued finitely additive set function $u$ and each $x \in X, \widehat{u \cdot x}=$ $\hat{u} \cdot x$ since then we have that $\hat{u}_{n}=G_{n}$. This follows since

$$
\widehat{u \cdot x}(f)=u \cdot x\left(A_{f}\right)=u\left(A_{f}\right) \cdot x=u(f) \cdot x .
$$

Lemma 3. The space $\mathrm{AC}(S, X, F)$ is a Banach space.

Proof. Since $\mathrm{BV}(S, X)$ is a Banach space, from Theorem 1 it is sufficient to show that $\operatorname{AC}(S, X, F)$ is closed in $\operatorname{BV}(S, X)$. Consider $G \in \mathrm{BV}(S, X)$ and $G_{n} \in \mathrm{AC}(S, X, F)$ where $\left\{G_{n}\right\}$ converges to $G$ in the BV-norm. Since each $G_{n}$ is the limit in the BV-norm of functions of the form $\Sigma_{i} G_{n, i} \cdot x_{n, i}$ where each $G_{n, i}<<F$, it follows that $G \in A C(S, F, X)$. 
Definition. Let $\left\{A_{1}, A_{2}, \cdots, A_{n}\right\}$ be a partition of $A$ by sets in $\Sigma$ and let $s=\sum_{i=1}^{n} \chi_{A_{i}} \cdot x_{i}$. Define

$$
V_{s}(E)=\int_{E} s d u_{F}
$$

for each $E$ in $\Sigma$; then clearly $V_{s}<<u_{F}$. The function $P_{s} \in \operatorname{AC}(S, F, X)$ which corresponds to $V_{s}$ from Theorem 1 will be called a polygonal function.

Lemma 4. The collection of polygonal functions is dense in $\operatorname{AC}(S, F, X)$.

Proof. Consider $G \in \operatorname{AC}(S, F, X)$ and $\epsilon>0$. There exists an $H=$ $\sum_{i=1}^{n} b_{i} \cdot x_{i}$, where $b_{i} \epsilon \mathrm{AC}(S, F)$ and $\|G-H\|_{\mathrm{BV}}<\epsilon$. Furthermore each $b_{i}=\hat{u}_{i}$, where $u_{i}<<u_{F}$. From the result of Darst [1], there exist simple functions $s_{i}$ such that $\left\|u_{i}-\int s_{i} d u_{F}\right\|<\epsilon$. Let $t=\Sigma s_{i} \cdot x_{i}$ and $V_{t}(E) \int_{E} t d u_{F}$; then if $P_{t}$ is the polygonal function which corresponds to $V_{t}$, we have

$$
\begin{aligned}
\left\|H-P_{t}\right\|_{\mathrm{BV}} & =\left\|\sum b_{i} \cdot x_{i}-P_{t}\right\|=\left\|\sum u_{i} \cdot x_{i}-V_{t}\right\| \\
& =\left\|\sum u_{i} \cdot x_{i}-\int \sum s_{i} \cdot x_{i} d u_{F}\right\| \leq \sum\left(\left\|u_{i}-\int \sum s_{i} d u_{F}\right\|\right)\left\|x_{i}\right\|
\end{aligned}
$$

which establishes the lemma.

Now to each $G$ in $\operatorname{AC}(S, X, F)$ we associate a special polygonal function which, in the case that $S$ is the set of characteristic functions on half open intervals, coincides with the usual idea of polygonal function (see [3]). Let $G \in$ $\operatorname{AC}(S, X, F)$, and let $Y$ be a finite subset of $S$. Let

$$
W_{Y, G}=\sum_{\sigma \in T} \frac{u_{G}(B(Y, \sigma))}{u_{F}(B(Y, \sigma))} \cdot \chi_{B(Y, \sigma)} \cdot
$$

Since $u_{F}(B(Y, \sigma))=0$ implies $u_{G}(B(Y, \sigma))=0$, we define the ratio to be zero in this case. Let $V_{Y, G}=\int W_{Y, G} d u_{F}$; then since $V_{Y, G}<<u_{F}$, we denote the corresponding polygonal function by $p G_{Y^{*}}$.

Lemma 5. The collection of all $p G_{Y}$ is dense in $\mathrm{AC}(S, X, F)$ in the BV-norm. In fact for $\epsilon>0$, there exists a finite subset $Y_{0}$ of $S$ such that if $Y \supset Y_{0}$ then $\left\|G-p G_{Y}\right\|<\epsilon$.

Proof. Let $\epsilon>0$; then there exists an $X$-valued simple function $s$ such that $\left\|u_{G}-V_{s}\right\|<\epsilon / 2$ since $u_{G}<<u_{F}$. If $s=\Sigma_{\sigma} \chi_{B(Z, \sigma)}$, then for each $B(Z, \sigma)$,

$$
V_{s}(B(Z, \sigma))=\int_{B(Z, \sigma)} s d u_{F}=u_{F}(B(Z, \sigma)) \cdot x_{\sigma} .
$$

Thus $x_{\sigma}=\left[1 / u_{F}(B(Z, \sigma))\right] \cdot V_{s}(B(Z, \sigma))$. Similarily, if $Z^{\prime} \supset Z$, we have

$$
s=\sum_{\sigma} \frac{1}{u_{F}\left(B\left(Z^{\prime}, \sigma\right)\right)} \cdot \chi_{B\left(Z^{\prime}, \sigma\right)}
$$


which we shall write as

$$
\sum_{\sigma} \frac{V_{s}\left(B\left(Z^{\prime}, \sigma\right)\right)}{u_{F}\left(B\left(Z^{\prime}, \sigma\right)\right)} \cdot \chi_{B\left(Z^{\prime}, \sigma\right)^{\bullet}}
$$

Now

$$
\begin{aligned}
\left\|V_{s}-u_{p G_{Z^{\prime}}}\right\| & \leq \int\left\|s-W_{Z^{\prime}, G}\right\| d u_{F} \\
& \leq \sum_{\sigma} \int_{B\left(Z^{\prime}, \sigma\right)}\left\|\frac{V_{s}\left(B\left(Z^{\prime}, \sigma\right)\right)-u_{G}\left(B\left(Z^{\prime}, \sigma\right)\right)}{u_{F}\left(B\left(Z^{\prime}, \sigma\right)\right)}\right\| d u_{F} \\
& =\sum_{\sigma}\left\|V_{s}\left(B\left(Z^{\prime}, \sigma\right)\right)-u_{G}\left(B\left(Z^{\prime}, \sigma\right)\right)\right\| \\
& \leq\left\|V_{s}-u_{G}\right\|<\epsilon / 2 .
\end{aligned}
$$

Hence the result follows from the triangular inequality and Theorem 1.

We will denote the space of all bounded linear maps from a Banach space $X$ to a Banach space $Y$ by $L(X, Y)$.

Definition. Let $K$ be a function defined on all sets of $B$-type with values in $L(X, Y)$. We say that $K$ is convex relative to $F$ if whenever $\left\{B\left(Z^{\prime}, \tau\right)\right\}, \tau \in T_{m^{\prime}}$, is a partition of $B(Z, \sigma)$, then

$$
K(B(Z, \sigma))=\sum_{\tau} \lambda_{\tau} K\left(B\left(Z^{\prime}, \tau\right)\right)
$$

where $\lambda_{\tau}=u_{F}\left(B\left(Z^{\prime}, \tau\right)\right) / u_{F}(B(Z, \sigma))$. The set function $K$ will be called bounded if $K$ is bounded in the $L(X, Y)$ norm over all sets of $B$-type. By $\|K\|$, we will mean the least upper bound of the bounds for $K$.

Definition. For $G: S \rightarrow X$ and $K$ convex, by the $v$-integral of $G$ with respect to $K$, we mean the limit, if it exists, of $\Sigma_{\sigma} K(B(Z, \sigma)) L(Z, \sigma) G$, where the limit is taken over the net of all finite subsets of $S$. We denote the integral when it exists by $v \int G d K$.

Lemma 6. All polygonal functions are v-integrable with respect to every convex and bounded $K$. In fact,

$$
v \int p_{S} d K=\sum_{\sigma} K(B(Z, \sigma)) L(Z, \sigma) p_{s}
$$

for all $Z \supset Z_{0}, Z_{0}$ some finite subset of $S$.

Proof. Suppose $s=\Sigma_{\sigma} B\left(Z_{0}, \sigma\right) \cdot x_{\sigma}$. Then $V_{s}(B(Z, \sigma))=u_{F}(B(Z, \sigma)) \cdot x_{\sigma}$ for all $Z \supset Z_{0^{\circ}}$. So $L(Z, \sigma) p_{s}=u_{F}(B(Z, \sigma)) \cdot x_{\sigma^{\circ}}$ Consider $Z_{0} \subset Z \subset Z^{\prime}$. Then $L\left(Z^{\prime}, \tau\right) p_{s}=u_{F}\left(B\left(Z^{\prime}, \tau\right)\right) \cdot x_{\tau}$ where $x_{\tau}=x_{\sigma}$ if $B\left(Z^{\prime}, \tau\right) \subset B(Z, \sigma)$. By convexity, $K\left(B\left(Z_{0}, \sigma\right)\right)=\Sigma \lambda_{\sigma} B\left(Z^{\prime}, \sigma\right)$ where $\lambda_{\sigma}=u_{F}\left(B\left(Z^{\prime}, \sigma\right)\right) / u_{F}\left(B\left(Z_{0}, \sigma\right)\right)$. 
Thus

$$
\sum_{\sigma} K\left(B\left(Z_{0}, \sigma\right)\right) L\left(Z_{0}, \sigma\right) p_{s}=\sum_{\tau} K\left(B\left(Z^{\prime}, \tau\right)\right) L\left(Z^{\prime}, \tau\right) p_{s^{*}}
$$

Theorem 2. Let $T$ be a linear operator from the space $\mathrm{AC}(S, X, F)$ into $Y$ which is continuous in the BV-norm. Then there exists a unique convex and bounded set function $K$, with values in $L(X, Y)$, sucb that every $G$ in $\mathrm{AC}(S, X, F)$ is $K$-integrable, and moreover

$$
T(G)=v \int G d K
$$

Furtbermore, $\|T\|=\|K\|$.

Conversely, if $K$ is any convex and bounded, $L(X, Y)$ valued set function, then each $G \in A C(S, X, F)$ is $K$-integrable and $v \int G d K$ defines a continuous linear operator from $\mathrm{AC}(S, X, F)$ into $Y$.

Proof. Let $Z$ be any finite subset of $S$ and let

$$
V_{Z, \sigma}(E)=\frac{u_{F}[B(Z, \sigma) \cap E]}{u_{F}(\dot{B(Z, \sigma))}}
$$

then $V_{Z, \sigma}$ is finitely additive and $V_{Z, \sigma}<<u_{F}$. Let $\psi_{Z, \sigma}$ be the corresponding function in $\operatorname{AC}(S, F)$. Define the function $K$ by the equation

$$
K(B(Z, \sigma)) \cdot x=T\left(\psi_{Z, \sigma} \cdot x\right) ;
$$

then

$$
\|K(B(Z, \sigma)) \cdot x\|_{Y}=\left\|T\left(\psi_{Z, \sigma} \cdot x\right)\right\|_{Y} \leq\|T\|\left\|\psi_{Z, \sigma} \cdot x\right\|_{\mathrm{BV}} \cdot
$$

Since $\left\|\psi_{Z, \sigma} \cdot x\right\|_{\mathrm{BV}}=\left\|V_{Z, \sigma} \cdot x\right\|=\left\|V_{Z, \sigma}\right\| \cdot\|x\| \leq\|x\|$, we have that $\|K\| \leq$ $\|T\| \cdot$ Now,

$$
V_{Z, G}(E)=\int_{E} \sum_{\sigma} \frac{u_{G}(B(Z, \sigma))}{u_{F}(B(Z, \sigma))} \cdot \chi_{B}(Z, \sigma) d u_{F}=\sum_{\sigma} u_{G}(B(Z, \sigma)) V_{Z, \sigma}(E) .
$$

Thus by Theorem 1, $p G_{Z}=\Sigma_{\sigma} L(Z, \sigma) G \psi_{Z, \sigma^{\circ}}$ From Lemma 5, we have

$$
\begin{aligned}
T(G) & =\lim _{Z} T\left(p G_{Z}\right)=\lim _{Z} T\left(\sum_{\sigma} L(Z, \sigma) G \psi_{Z, \sigma}\right) \\
& =\lim _{Z} K(B(Z, \sigma)) L(Z, \sigma) G=v \int G d K .
\end{aligned}
$$

Al so 


$$
\begin{aligned}
\|T\| & \geq \sup _{\|x\| \leqslant 1}\left\|T\left(\psi_{Z, \sigma} \cdot x\right)\right\|_{Y} \\
& =\sup _{\|x\| \leqslant 1}\|K(B(Z, \sigma)) \cdot x\|_{Y}=\|K(B(Z, \sigma))\|_{L(X, Y)^{\bullet}}
\end{aligned}
$$

Hence $\|T\|=\|K\|$.

Conversely suppose that $K$ is a bounded, convex, $L(X, Y)$ valued set function. Then

$$
\left\|v \int p G_{Z_{1}} d K-v \int p G_{Z_{2}} d K\right\| \leq\left\|p G_{Z_{1}}-p G_{Z_{2}}\right\|\|K\|
$$

Since $Y$ is complete this shows that $G$ is $K$-integrable and moreover that

$$
v \int G d K=\lim _{z} v \int p G_{Z} d K
$$

We now define the concept of a Lipschitz function and characterize the space of all such functions in terms of convex and bounded set functions.

Definition. Let $g$ be a real valued function defined on $S$. Then $g$ is called Lipschitz with respect to $F$ if there exists a constant $P$ such that $|L(z, \sigma) g|<$ $P L(z, \sigma) F$ for all sets $B(x, \sigma)$. We denote this space of functions by $\operatorname{Lip}(F)$.

Definition. By the space $\operatorname{Lip}(X, F)$ we mean all functions $G \in \mathrm{BV}(S, X, F)$ which are approximable in the BV-norm by functions of the form $\sum_{i=1}^{n} g_{i} \cdot x_{i}$, where $x_{i} \in X$ and $g_{i} \in \operatorname{Lip}(F)$ for $i=1,2, \cdots, n$.

We now want to give a characterization of the space $\operatorname{Lip}(X, F)$ in terms of convex and bounded set functions. For this purpose we introduce a special class of convex and bounded set functions which we denote by $M_{C}(X, F)$.

Definition. Let $K$ be a convex and bounded $X$-valued set function. We say that $K \in M_{C}(X, F)$ if and only if, for each $\epsilon>0$, there are finite collections $\left\{K_{1}, K_{2}, \cdots, K_{n}\right\}$ and $\left\{x_{1}, x_{2}, \cdots, x_{n}\right\}$, where each $K_{i}$ is scalar, convex, and bounded and each $x_{i} \in X$, and such that

$$
\sum u_{F}\left(B_{j}\right)\left\|K\left(B_{j}\right)-\sum_{i=1}^{n} K_{i}\left(B_{j}\right) \cdot x_{i}\right\|_{X}<\epsilon
$$

for all partitions $\left\{B_{j}\right\}$ of $A$ into sets of $B$-type. Clearly, $M_{C}(X, F)$ is a linear space.

Theorem 3. The spaces $M_{C}(X, F)$ and $\operatorname{Lip}(X, F)$ are linearly isomorphic.

Proof. Consider $H \in \operatorname{Lip}(X, F)$ and $\epsilon>0$, then there exists a finite set $\left\{b_{1}, b_{2}, \ldots, b_{n}\right\}$ where each $b_{i} \in \operatorname{Lip}(F)$ and a finite set $\left\{x_{1}, x_{2}, \ldots, x_{n}\right\}$, $x_{i} \in X$, such that $\left\|H-\sum_{i=1}^{n} b_{i} \cdot x_{i}\right\|_{\mathrm{B} \mathrm{V}}<\epsilon$. Let $u_{H}$ correspond to $H$ and define 
$K_{H}$ by the equation $K_{H}(B)=u_{H}(B) / u_{F}(B)$, and, if $m_{i}$ corresponds to $b_{i}$, define $k_{i}$ by the equation $k_{i}(B)=u_{i}(B) / u_{F}(B)$, for each $i$, for all sets $B$ of $B$-type. It follows that the set functions $K_{H}, K_{1}, \ldots, K_{n}$ are all convex and bounded. We now show that $K_{H} \in M_{C}(X, F)$. Let $\left\{B_{j}\right\}$ be a partition of $A$ into sets of the B-type. Then

$$
\sum_{j} u_{F}\left(B_{j}\right)\left\|K_{H}\left(B_{j}\right)-\sum_{i=1}^{n} k_{i}\left(B_{j}\right) \cdot x_{i}\right\|=\sum_{j}\left\|u_{H}\left(B_{j}\right)-\sum_{i=1}^{n} m_{i}\left(B_{j}\right) \cdot x_{i}\right\|<\epsilon .
$$

Conversely, consider $K \in M_{C}(X, F)$. Then for $\epsilon>0$ there exists $\left\{K_{1}, K_{2}\right.$, $\left.\ldots, K_{n}\right\}$ and $\left\{x_{1}, x_{2}, \ldots, x_{n}\right\}$ such that

$$
\sum_{j} u_{F}\left(B_{j}\right)\left\|K\left(B_{j}\right)-\sum_{i=1}^{n} K_{i}\left(B_{j}\right) \cdot x_{i}\right\|<\epsilon
$$

for all partitions $\left\{B_{j}\right\}$ of $A$ into sets of $B$-type. If we define $u_{K}(B)=u_{F}(B) K(B)$ and $m_{i}(B)=u_{F}(B) K_{i}(B)$, then it is easy to check that $u_{K}$ and the $m_{i}$ 's are finitely additive and absolutely continuous with respect to $F$. Let $H_{K}$ correspond to $u_{K}$ where $H_{K} \in A C(S, X, F)$ and $b_{i}$ correspond to $m_{i}$ where $b_{i} \in \operatorname{AC}(S, F)$; then $\left\|H_{K}-\sum_{i=1}^{n} b_{i} x_{i}\right\|_{\mathrm{B} \mathrm{V}}<\epsilon$. Now the maps $K \rightarrow H_{K}$ and $H \rightarrow K_{H}$ are inverses of one another. Consequently, the theorem is shown since linearity is immediate.

Remark. It should be pointed out that the above characterization is rather different from the scalar case as in [1]. While the map $H \rightarrow K_{H}$ was straightforward in the scalar case, we have seen that in our vector setting a weightedtype of variation is needed to define the map.

\section{BIBLIOGR APHY}

1. Richard A. Alò and Andre de Korvin, Functions of bounded variation on idempotent semigroups, J. Math. Anal. Appl. 194 (1971), 1-11.

2. R. B. Darst, A decomposition of finitely additive set functions, J. Reine Angew. Math. 210 (1962), 31-37. MR 25 \#1257.

3. A. de Korvin and R. J. Easton, A representation theorem for $\mathrm{AC}\left(R^{n}\right)$, Scripta Math. (to appear).

4. N. Dinculeanu, Vector measures, Internat. Series of Monographs in Pure and Appl. Math., vol. 95, Pergamon Press, Oxford; VEB Deutscher Verlag der Wissenschaften, Berlin, 1967. MR 34 \#6011b.

5. N. Dunford and J. T. Schwartz, Linear operators. I: General theory, Pure and Appl. Math., vol. 7, Interscience, New York, 1958. MR 22 \#3302.

6. J. Edwards and S. Wayment, Representations for transformations continuous in the BV norm, Trans. Amer. Math. Soc. 154 (1971), 251-265. MR 43 \#466.

7. T. Hildebrandt, Linear continuous functionals on the space (BV) with weak topologies, Proc. Amer. Math. Soc. 17 (1966), 558-664. MR 33 \#1710. 
8. S. E. Newman, Measure algebras and functions of bounded variation on idempotent semigroups, Bull. Amer. Math. Soc. 75 (1969), 1396-1400. MR 40 \#4778.

9. G.-C. Rota, On the foundations of combinatorial theory: I. Theory of Möbius functions, Z. Wahrscheinlichkeitstheorie und Verw. Gebiete 2 (1964), 340-368. MR 30 \#4688.

DEPARTMENT OF MATHEMATICS, CARNEGIE-MELLON UNIVERSITY, PITTSBURGH, PENNSYLVANIA 15213

DEPARTMENT OF MATHEMATICS, INDIANA STATE UNIVERSITY, TERRE HAUTE, INDIANA 47809 